\title{
Spin and dipole order in geometrically frustrated mixed-valence manganite $\mathrm{Pb}_{3} \mathrm{Mn}_{7} \mathrm{O}_{15}$
}

\author{
S. A. Ivanov'1,2 • A. A. Bush ${ }^{3} \cdot$ M. Hudl ${ }^{4}$ - A. I. Stash ${ }^{1}$ - G. André ${ }^{5}$. \\ R. Tellgren ${ }^{6}$ V. M. Cherepanov ${ }^{7}$ - A. V. Stepanov ${ }^{3}$ K. E. Kamentsev ${ }^{3}$.

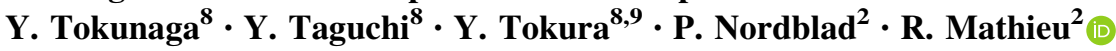

Received: 21 May 2016/Accepted: 15 July 2016/Published online: 21 July 2016

(c) The Author(s) 2016. This article is published with open access at Springerlink.com

\begin{abstract}
The structural, magnetic, and dielectric properties of $\mathrm{Pb}_{3} \mathrm{Mn}_{7} \mathrm{O}_{15}$ have been investigated using highquality single crystals. $\mathrm{Pb}_{3} \mathrm{Mn}_{7} \mathrm{O}_{15}$ adopts a pseudohexagonal orthorhombic structure, with partially filled Kagomé layers connected by ribbons of edge-sharing $\mathrm{MnO}_{6}$ octahedra and intercalated $\mathrm{Pb}$ cations. There are 9 inequivalent sites in the structure for the $\mathrm{Mn}$ ions, which exist both as $\mathrm{Mn}^{3+}$ and $\mathrm{Mn}^{4+} \cdot \mathrm{Pb}_{3} \mathrm{Mn}_{7} \mathrm{O}_{15}$ undergoes an antiferromagnetic transition below $\mathrm{T}_{\mathrm{N}} \sim 67 \mathrm{~K}$, with significant geometric frustration. Neutron powder diffraction on crushed single crystals allowed us to determine the lowtemperature antiferromagnetic magnetic structure. We
\end{abstract}

Electronic supplementary material The online version of this article (doi:10.1007/s10854-016-5387-3) contains supplementary material, which is available to authorized users.

R. Mathieu

roland.mathieu@angstrom.uu.se

1 Center of Materials Science, Karpov Institute of Physical Chemistry, Vorontsovo pole, 10, Moscow, Russia 105064

2 Department of Engineering Sciences, Uppsala University, Box 534, SE-751 21 Uppsala, Sweden

3 Moscow Technological University, Moscow, Russia 119434

4 Department of Physics, Stockholm University, SE-106 91 Stockholm, Sweden

5 Laboratoire Leon Brillouin, CEA, Saclay, France

6 Department of Chemistry - Ångström Laboratory, Uppsala University, Box 538, SE-751 21 Uppsala, Sweden

7 National Research Centre "Kurchatov Institute", Moscow, Russia 123182

8 RIKEN Center for Emergent Matter Science (CEMS), Wako 351-0198, Japan

9 Department of Applied Physics, University of Tokyo, Tokyo 113-8656, Japan discuss the magnetic interaction pathways in the structure and possible interplay between the structural distortions imprinted by the lone-electron pair of $\mathrm{Pb}^{2+}$ cations and $\mathrm{Mn}^{3+} / \mathrm{Mn}^{4+}$ charge ordering.

\section{Introduction}

Manganites are materials with properties relevant for both fundamental research and practical applications, owing to the cross-correlation of their charge, spin, orbital and lattice degrees of freedom. Certain manganites have for example been found to display colossal magnetoresistance [1-4], multiferroicity and magneto(di)electric effects [5-7]. Lone-pair $\mathrm{Pb}^{2+}$ cations may induce polar distortions that couple charge and spin orders. In this context, oxide compounds from the phase diagram of the PbO-MnO-O system, combining $\mathrm{Pb}$ and $\mathrm{Mn}$ cations, are very interesting as they may display concomitant and coupled dielectric and magnetic orders. At atmospheric pressure, the following phases of the $\mathrm{PbO}-\mathrm{MnO}-\mathrm{O}$ system have been established (see Fig. 1): $\mathrm{Pb}_{1+\mathrm{x}} \mathrm{Mn}_{8} \mathrm{O}_{16}$ hollandite-type structure [8, 9], $\mathrm{Pb}_{3} \mathrm{Mn}_{7} \mathrm{O}_{15}$ [8, 10-13, 15-18], $\mathrm{PbMn}_{2} \mathrm{O}_{4}$ [11, 12], and $\mathrm{Pb}_{4} \mathrm{Mn}_{9} \mathrm{O}_{20}$ [19] with close packed structures; $\mathrm{Pb}_{0.25} \mathrm{MnO}_{2}$, $\mathrm{Pb}_{0.25} \mathrm{MnO}_{2-\mathrm{x}}(\mathrm{x}=0,01)$ [13] with unknown structures, as well as $\mathrm{Pb}_{2} \mathrm{MnO}_{4}[20,21]$. Utilizing high pressure $(>7$ $\mathrm{GPa})$ and high temperature $\left(\mathrm{T}>800^{\circ} \mathrm{C}\right)$ techniques the phases $\mathrm{PbMnO}_{2.75}$ [22], $\mathrm{PbMnO}_{3}$ [23], $\mathrm{Pb}_{13} \mathrm{Mn}_{9} \mathrm{O}_{25}$ [24], and $\mathrm{PbMn}_{7} \mathrm{O}_{12}$ [25] with perovskite-based structure have been obtained.

$\mathrm{Pb}_{3} \mathrm{Mn}_{7} \mathrm{O}_{15}$ (PMO) is a mixed valence manganite $\left(\mathrm{Mn}^{3+} / \mathrm{Mn}^{4+}\right)$, whose structural [14-17], magnetic and dielectric [10-13, 26-30] properties have been studied in detail. The material is a dielectric relaxor, and becomes 

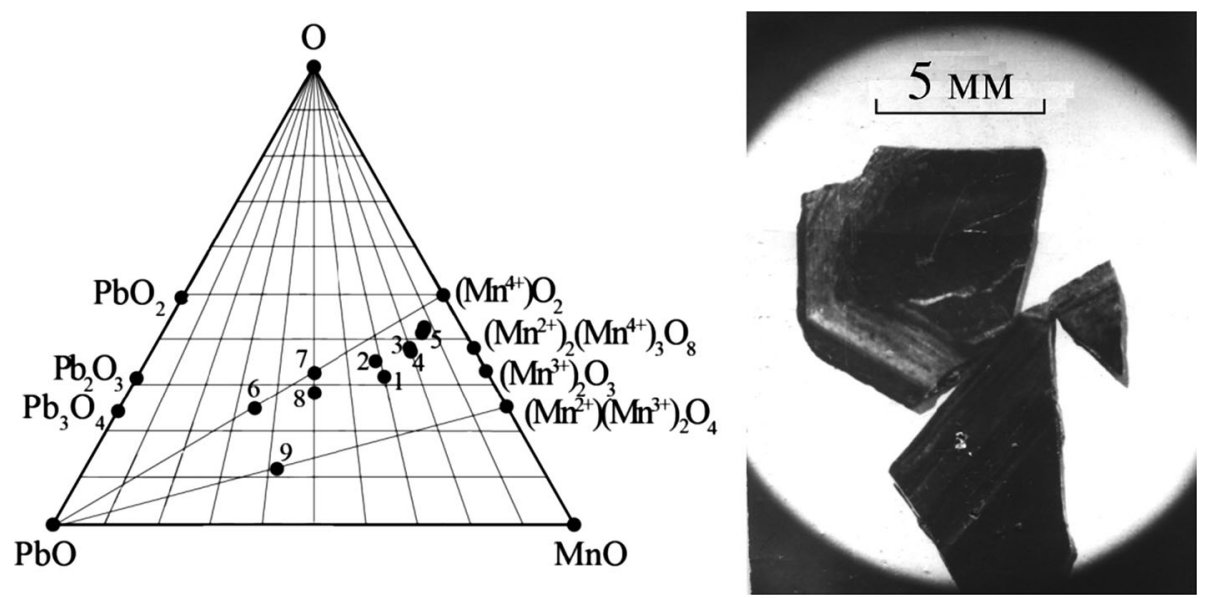

Fig. 1 (Left) known phases of the PbO-MnO-O system: $1 \mathrm{~Pb}_{3} \mathrm{Mn}_{7}$ $\mathrm{O}_{15}=\mathrm{Pb}_{3}\left(\mathrm{Mn}^{3+}\right)_{4}\left(\mathrm{Mn}^{4+}\right)_{3} \mathrm{O}_{15}$ [8, 10-17], $2 \mathrm{~Pb}_{4}\left(\mathrm{Mn}^{3.56+}\right)_{9} \mathrm{O}_{20}$ [18], $3 \mathrm{~Pb}_{0,25}\left(\mathrm{Mn}^{3.5+}\right) \mathrm{O}_{2}$ [13], $4 \mathrm{~Pb}_{0.25} \mathrm{MnO}_{1.99}$ [13], $5 \mathrm{~Pb}_{1+\mathrm{x}} \mathrm{Mn}_{8} \mathrm{O}_{16}=$ $\mathrm{Pb}_{1+\mathrm{x}}\left(\mathrm{Mn}^{3+}\right)_{2+2 \mathrm{x}}\left(\mathrm{Mn}^{4+}\right)_{6-2 \mathrm{x}} \mathrm{O}_{16}[8,9], 6 \mathrm{~Pb}_{2}\left(\mathrm{Mn}^{4+}\right) \mathrm{O}_{4}[19,20], 7$

antiferromagnetic below $T_{N} \sim 67 \mathrm{~K}$. However, there is a long-standing controversy about the structure of this material, and its microscopic magnetic structure is yet unknown. The first structure determination of PMO was reported by Darriet et al. [14] who concluded that the symmetry at $295 \mathrm{~K}$ is orthorhombic. The orthorhombic description was later supported by Marsh et al. [31]. Le Page et al. [15] subsequently argued that this structure is really hexagonal. More recently, Volkov et al. [27] reported as well an hexagonal structure, s.g. $P 6_{3} / \mathrm{mcm}$, similar to that the mineral Zenzenite $\mathrm{Pb}_{3}(\mathrm{Fe}, \mathrm{Mn})_{7} \mathrm{O}_{15}$ [32]. However a high resolution study by Rasch et al. [16] using synchrotron data demonstrated instead an orthorhombic symmetry. In another study, Volkov et al. [17] and Kimber [28] also reported an orthorhombic Pnma structure for PMO at $298 \mathrm{~K}$, albeit noting that it was close to a structural phase transition, with a pseudo-hexagonal cell at $298 \mathrm{~K}$, evolving into an hexagonal structure at higher temperatures $[17,28]$. Stabilization of hexagonal structure at room-temperature after thermal treatment is not possible [17]. Interestingly, the hexagonal structure may also be stabilized by doping, e.g. by replacing only $5 \%$ of $\mathrm{Mn}$ by $\mathrm{Fe}^{3+}$ [29]—like Zenzenite mineral. Doping by $5 \% \mathrm{Ga}^{3+}$ or $\mathrm{Ge}^{4+}$ keeps the structure orthorhombic. Relatively small changes of the magnetic properties were observed for low doping levels [33].

There remain unsolved issues as to both the nuclear and the magnetic structure as well as the dielectric and macroscopic magnetic properties of PMO. In this article, the structural, thermal, magnetic and dielectric properties of stoichiometric single crystals of $\mathrm{Pb}_{3} \mathrm{Mn}_{7} \mathrm{O}_{15}$ are investigated. Powder X-ray and neutron diffraction data recorded on crushed single-crystals confirm a pseudo-hexagonal orthorhombic structure, and allow us to determine the
$\mathrm{Pb}\left(\mathrm{Mn}^{4+}\right) \mathrm{O}_{3}$ [21], $8 \mathrm{~Pb}\left(\mathrm{Mn}^{3.5+}\right) \mathrm{O}_{2.75}$ [22], $9 \mathrm{~Pb}_{13} \mathrm{Mn}_{9} \mathrm{O}_{25}=\mathrm{Pb}_{13}$ $\left.\left(\mathrm{Mn}^{2.36(6)+}\right)_{9} \mathrm{O}_{25}\right)$ [23]. (Right) photograph of a typical single crystal of $\mathrm{Pb}_{3} \mathrm{Mn}_{7} \mathrm{O}_{15}$

magnetic structure of the low-temperature antiferromagnetic state of $\mathrm{Pb}_{3} \mathrm{Mn}_{7} \mathrm{O}_{15}$.

\section{Experimental}

High-quality single crystals of $\mathrm{Pb}_{3} \mathrm{Mn}_{7} \mathrm{O}_{15}$ were grown in $\mathrm{Pt}$ crucibles in a $\mathrm{PbO}$ flux in an intimate mixture of 9PbO- $\mathrm{Mn}_{2} \mathrm{O}_{3}$, as described in Bush et al. [12]. Excess flux was dissolved in $\mathrm{HNO}_{3}$. Shiny, opaque, black and platelike crystals of dimensions up to $2 \times 10 \times 10 \mathrm{~mm}^{3}$ were obtained. The crystals occurred with hexagonal habits, showing the main forms as $\{0001\},\{10-12\}$ including also poorly developed $\{11-21\}$ forms. For Mössbauer studies $2 \% \mathrm{Fe}$-doped $\mathrm{Pb}_{3}\left(\mathrm{Mn}_{0.98} \mathrm{Fe}_{0.02}\right)_{7} \mathrm{O}_{15}$ single crystals were grown under similar conditions adding $2 \mathrm{~mol} \%{ }^{57} \mathrm{Fe}_{2} \mathrm{O}_{3}$ (instead of the same amount of $\mathrm{Mn}_{2} \mathrm{O}_{3}$ ). Phase purity was checked by powder X-ray diffraction.

The phase stability of PMO was investigated by thermogravimetry analysis, and the cations stoichiometry by energy-dispersive spectroscopy (EDS). Several iodometric titrations were made on $\mathrm{Pb}_{3} \mathrm{Mn}_{7} \mathrm{O}_{15+\delta}$ single crystals to determine the oxygen content. Second harmonic generation (SHG) measurements at room temperature evidenced the centrosymmetric character of the crystals.

Crystal and magnetic structures were investigated by single crystal and powder X-ray diffraction (XRPD), and neutron powder diffraction on crushed single-crystals (NPD; data collected at the LLB, Saclay, France). Refinements of the XRPD and NPD data were performed by the Rietveld method using the FULLPROF software [34]. An analysis of coordination polyhedra of cations was performed using the IVTON software [35]. 
The Mössbauer spectroscopy measurements were performed using a flow type helium cryostat and a conventional constant acceleration type spectrometer in transmission geometry with a ${ }^{57} \mathrm{Co}(\mathrm{Cr})$ source, which had an activity of $5 \mathrm{mCi}$ and was kept at $300 \mathrm{~K}$. The Mössbauer spectra were analyzed using the SPECTR program from the MSTools package [36] in the Lorentz line approximation. Isomer shifts were determined in relation to the spectrum centroid of $\alpha$-Fe.

Magnetic, thermal, and dielectric properties of PMO were investigated on single crystals. Magnetization data was recorded as a function of temperature and magnetic field on an MPMS squid magnetometer and a PPMS system with VSM option from Quantum Design Inc. In the same setup, heat capacity data was collected using a relaxation method, and magneto(di)electric and pyroelectric measurements were performed under magnetic fields using external LCR meter and electrometer.

The Supplemental Material gives further experimental details.

\section{Results and discussion}

The stoichiometry of the single crystals was investigated using EDS and iodine titration. A $\mathrm{Pb}: \mathrm{Mn}$ cation ratio of $1: 2.335(2)$ was found. For the crystal selected for X-ray single crystal diffraction and study of physical properties, the average oxygen index $\delta$ was positive and of the order of $0.02(1)$. In the case of the powder sample which was used for neutron powder diffraction studies, an additional batch of single crystals was used and the resulting $\delta$ was of the order of $-0.03(1)$.

Single-crystal XRD data at $295 \mathrm{~K}$ indicates a hexagonal structure s.g. $\mathrm{PC}_{3} / \mathrm{mcm}$, with lattice parameters: $\mathrm{a}=9.979(1) \AA, \mathrm{c}=13.598(3) \AA$. The main structural results are presented in Table 1 and Table SM1 in the Supplemental Materials. On the other hand, the best refinements of powder XRD data on crushed single crystals are obtained for an orthorhombic Pnma structure (see Fig. 2). Comparison of hexagonal and orthorhombic models derived from XRPD data gave worse results for first one $\left(R_{p}, R_{w p}, R_{B}=2.39,3.46\right.$ and 4.79 respectively for hexagonal model, and 1.96, 2.73, and 4.03 for orthorhombic one) and the peak shape of several reflections clearly showed a splitting (see inset of Fig. 2); thus confirming that the space group of PMO is Pnma or Pn2 a. Centrosymmetric model Pnma was selected based on SHG results. Conversely, high-resolution diffraction studies using X-ray single crystal data showed no detectable metric deviation from a hexagonal lattice. Refinement of the same experimental set of intensities based on orthorhombic model (with increased number of refined parameters) gave us a worse agreement between the measured data sets and calculations $\left(R_{B}=4.6\right.$ vs. 3.96). Crystallographic results for PMO and associated polyhedral analysis are summarized in Tables 1 and 2. As illustrated in Fig. 3, the orthorhombic and hexagonal structures are closely related, and may be compared, considering that the hexagonal basal plane $(a, b)$ corresponds to the $(b, c)$ plane in the orthorhombic structure $\left(\mathrm{a}_{\text {ortho }} \approx \mathrm{c}_{\text {hexa }}\right.$, $\mathrm{b}_{\text {ortho }} \approx 2 \mathrm{a}_{\text {hexa }}+\mathrm{b}_{\text {hexa }}$, and $\mathrm{c}_{\text {ortho }} \approx \mathrm{b}_{\text {hexa }}$ ).

The existence of pseudo-symmetry in the PMO crystal structure is indicative of a distorted structure of higher symmetry. If the distortion is small enough, it can be expected that the crystal acquires this more symmetric configuration at a higher temperature. At high temperatures, hexagonal symmetry with space group $P 6_{3} / \mathrm{mcm}$ is indeed observed [17, 28], while as the temperature decreases, a distortion occurs and the structure becomes orthorhombic, with space group Pnma, i.e. a subgroup of $\mathrm{PG}_{3} / \mathrm{mcm}$.

The displacive phase transition from $\mathrm{Pb}_{3} / \mathrm{mcm}$ to Pnma in PMO can be quantified in terms of the amplitudes of the condensing modes, which generate the observed static distortion. The character and number of these modes can be derived by the group-subgroup relation between the hexagonal and the orthorhombic phases. The amplitudes, character, and number of condensing modes were derived using the AMPLIMODES software [37, 38]. Given the high- and the low-symmetry structures, AMPLIMODES determines the atomic displacements that relate them, defines a basis of symmetry-adapted modes, and calculates the amplitudes and polarization vectors of the distortion
Table 1 Summary of the results of the structural refinements of the $\mathrm{Pb}_{3} \mathrm{Mn}_{7} \mathrm{O}_{15}$ samples using single crystal and powder XRD data and NPD data (see Table SM1 for atomic coordinates and quality factors for the refinements)

\begin{tabular}{llllll}
\hline Experiment & Crystal & Powder & & \\
\cline { 3 - 6 } & X-ray & X-ray & Neutron & & \\
\hline T, K & 295 & 295 & 295 & 70 & 1.8 \\
a $[\AA]$ & $9.979(1)$ & $13.6127(2)$ & $13.598(1)$ & $13.546(1)$ & $13.526(1)$ \\
b $[\AA]$ & $9.979(1)$ & $17.3437(4)$ & $17.331(2)$ & $17.262(2)$ & $17.281(3)$ \\
c $[\AA]$ & $13.598(3)$ & $10.0226(2)$ & $10.009(1)$ & $9.926(1)$ & $9.9219(8)$ \\
s.g. & P63/mcm & Pnma & Pnma & Pnma & Pnma \\
\hline
\end{tabular}


Fig. 2 Final Rietveld plot of the refined XRPD data collected at $295 \mathrm{~K}$. The upper curves represent the collected data and calculated pattern; the lower curve represent the different of the two upper curves. Tick marks show positions of Bragg reflections. The insert illustrates the splitting of the hexagonal (110) reflection $\left(\mathrm{Pb}_{3} / \mathrm{mcm}\right)$ into orthorhombic (002) and (031) doublet $($ Pnma $)$ at $\mathrm{T}=295 \mathrm{~K}$

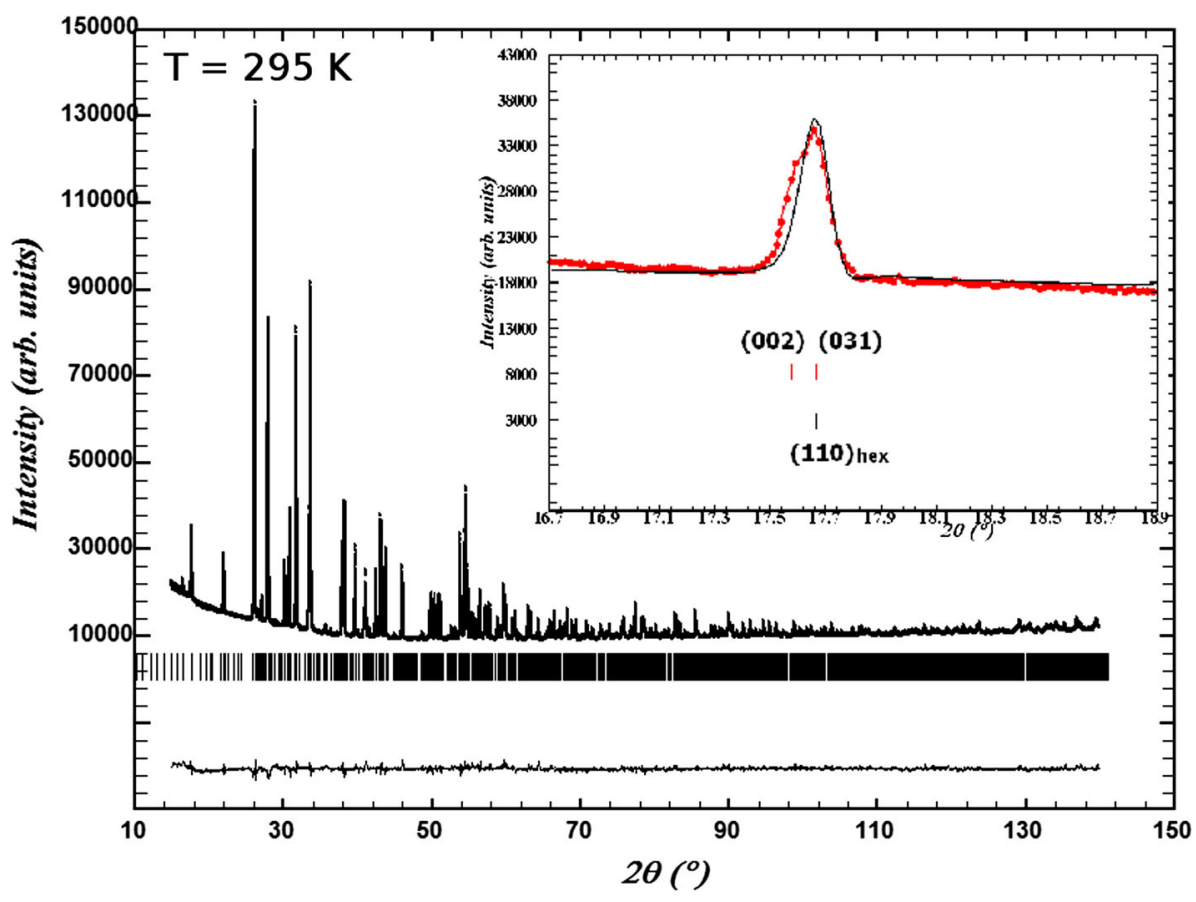

Table 2 Polyhedral analysis of $\mathrm{Pb}_{3} \mathrm{Mn}_{7} \mathrm{O}_{15}$ crystals at $295 \mathrm{~K}$ $(\delta$ - cation shift from centroid, $\xi$ - average $\mathrm{Mn}-\mathrm{O}$ and $\mathrm{Pb}-\mathrm{O}$ and bond distances, $\mathrm{V}$ polyhedral volume, $\Delta-$ polyhedral volume distortion)

\begin{tabular}{lllllrll}
\hline Cation & Number & c.n. & $\delta(\AA)$ & $\xi(\AA)$ & $\mathrm{V}\left(\AA^{3}\right)$ & \multicolumn{1}{l}{$\Delta$} & Valence \\
\hline Mn & 1 & 6 & 0.053 & $1.958 \pm 0.148$ & 9.65 & 0.016 & 3.59 \\
& 2 & & 0.193 & $1.909 \pm 0.137$ & 9.13 & 0.034 & 3.77 \\
& 3 & & 0.014 & $1.950 \pm 0.175$ & 9.25 & 0.053 & 3.68 \\
& 4 & & 0.174 & $1.992 \pm 0.147$ & 10.22 & 0.028 & 3.16 \\
& 5 & & 0.192 & $2.075 \pm 0.341$ & 11.18 & 0.037 & 3.09 \\
& 6 & & 0 & $1.876 \pm 0.123$ & 8.67 & 0.016 & 4.08 \\
& 7 & & 0 & $2.076 \pm 0.057$ & 11.54 & 0.032 & 2.82 \\
& 8 & & 0.196 & $1.926 \pm 0.190$ & 9.02 & 0.068 & 3.83 \\
& 9 & & 0.149 & $1.908 \pm 0.118$ & 8.98 & 0.038 & 4.11 \\
& 1 & 10 & 0.645 & $2.816 \pm 0.364$ & 45.38 & 0.174 & 2.18 \\
& 2 & 8 & 0.550 & $2.897 \pm 0.567$ & 37.64 & 0.207 & 2.16 \\
& 3 & 8 & 0.504 & $2.891 \pm 0.446$ & 36.07 & 0.224 & 1.91 \\
& 4 & 9 & 0.675 & $2.788 \pm 0.461$ & 35.66 & 0.316 & 2.31 \\
\hline
\end{tabular}

modes. The results of this decomposition are reported in Table SM2 in the Supplemental Materials. The structural transition is associated with three main modes. These primary $G M 1+, G M 5+$ and $M 2-$ modes have the largest amplitudes with values of $0.44,0.76$ and $1.36 \AA$, respectively. We find a total distortion amplitude (the amplitude of the sum of all condensing mode vectors) of $2.29 \AA$ with atomic displacements ranging from 0.05 to $0.36 \AA$.

Figure 4 shows the temperature dependence of the dielectric parameters $\varepsilon$ and $\tan \delta$ recorded under magnetic fields up to $9 \mathrm{~T}$ ( $\mathbf{E}$ along the hexagonal c-axis, i.e. [001], $\mathbf{H}$ along [1-10] in the (a, b)-plane). $\varepsilon$ increases sharply at around $100-200 \mathrm{~K}$ depending on the frequency, qualitatively in a similar way as earlier reported [30] (note that the crystals dimensions - area and thickness after polishing-were only roughly estimated in our experiments, and that other experiments suggest $\varepsilon \sim 30$ at low temperatures, akin to that estimated in [30]). The sharp steps in $\varepsilon$ correspond to well-defined maxima in the $\tan \delta$ curves. Fitting the observed relaxation to the Arrhenius behavior $\tau=\tau_{\mathrm{o}} \exp \left(\mathrm{E}_{\mathrm{a}} / \mathrm{k}_{\mathrm{B}} \mathrm{T}\right)[40,41],\left(\mathrm{k}_{\mathrm{B}}\right.$ is the Boltzmann constant, $\tau=1 / 2 \pi \mathrm{f}$ ) yields an activation energy $\mathrm{E}_{\mathrm{a}} \sim 0.18 \mathrm{eV}$ and $\mathrm{a}$ relaxation time $\tau_{\mathrm{o}} \sim 10^{-11} \mathrm{~s}$. The derived relaxation parameters $\left(\mathrm{Ea}, \tau_{\mathrm{o}}\right)$ which were found for PMO are characteristic of polaron relaxation [30]. As seen in Fig. 4, some magnetic field dependence, albeit weak, is observed in $\varepsilon$ and 


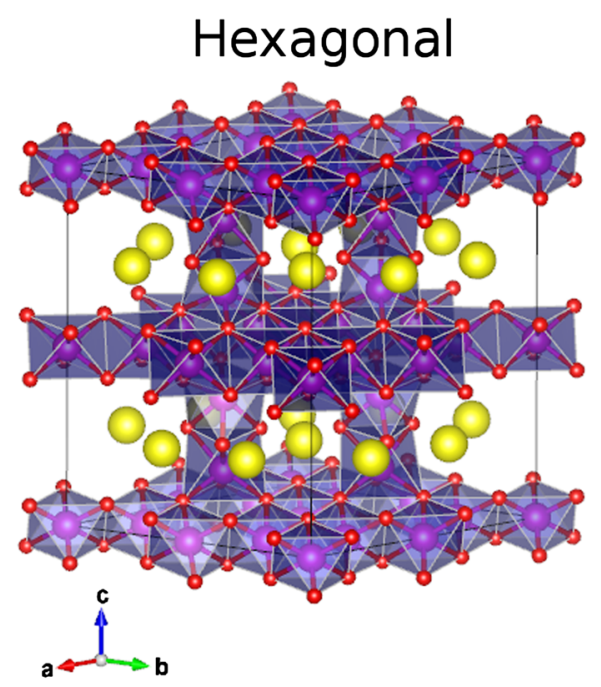

\section{Orthorhombic}
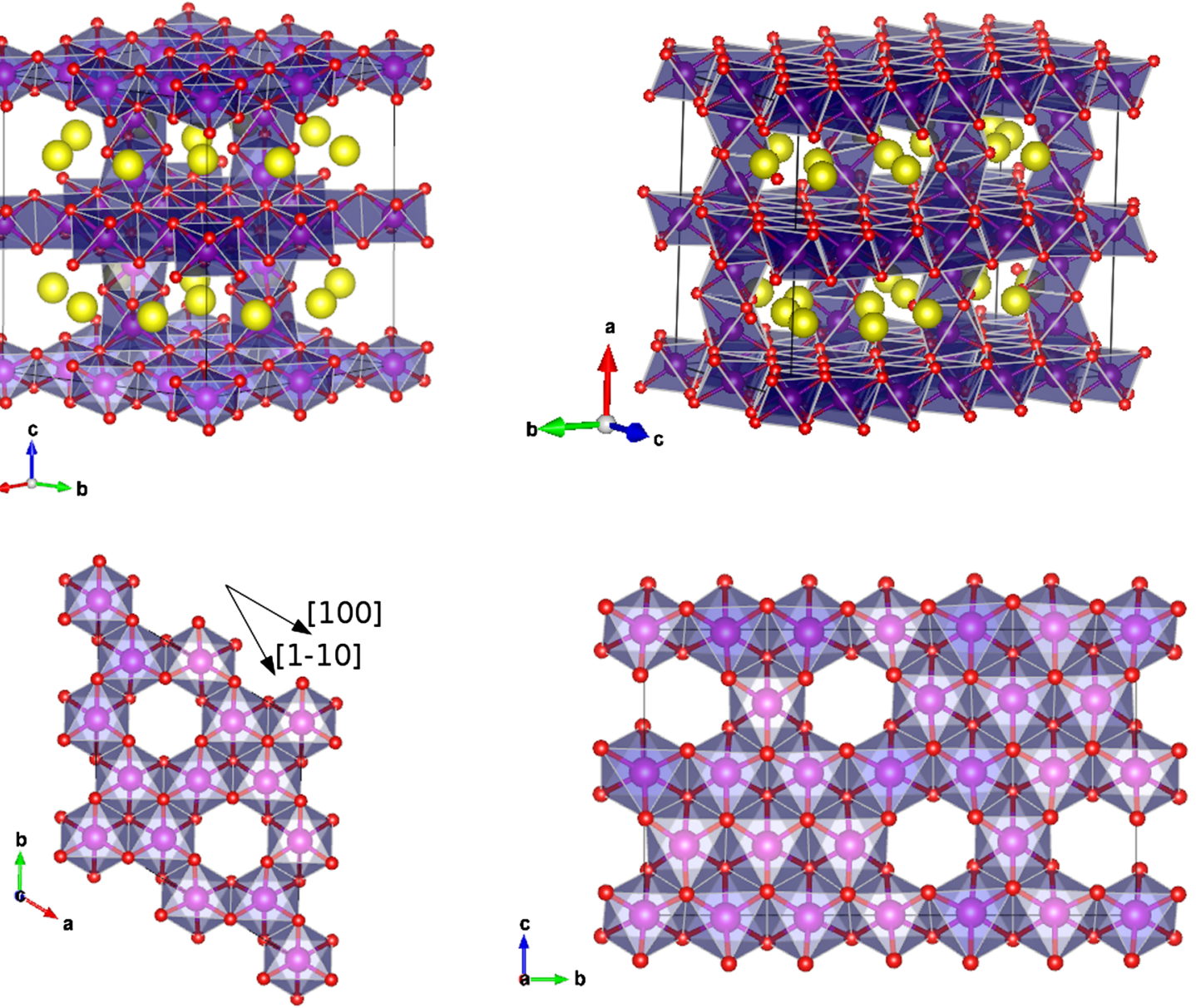

Fig. 3 Polyhedral representations of the crystal structure in three dimensions and in the basal plane for hexagonal (left, $(a, b)$-plane $)$ and orthorhombic (right, $(b, c)$-plane) structures; drawn using VESTA

[39]. In the hexagonal case, the two directions [100] and [1-10] in the basal plane considered for magnetic and dielectric measurements are indicated

$\tan \delta$ curves. No magneto-(di)electric effects could be observed, as in the earlier dielectric study of PMO reported in [30].

The Mössbauer spectra of the Fe-doped PMO sample registered just above $\mathrm{T}_{\mathrm{N}}(70 \mathrm{~K})$ and at low temperatures $(10 \mathrm{~K})$ are shown in Fig. 5. The latter spectrum demonstrates a typical broadened sextet of lines showing the presence of a magnetic order while the latter indicates a typical paramagnetic doublet and hence shows the absence of both long and short range magnetic order. The spectrum at $10 \mathrm{~K}$ was fitted using a superposition of two sextets and a singlet (Fig. 5, lower panel). The spectrum at $\mathrm{T}=70 \mathrm{~K}$ was fitted using a superposition of two paramagnetic doublets and a singlet (Fig. 5, upper panel). The main parameters extracted from the Mössbauer spectra, isomer shift-IS with respect to metallic iron, quadrupole splitting-QS, the effective magnetic field $\mathrm{H}$ and the relative area $\mathrm{A}$ of partial spectra, are collected in Table 3. From the obtained parameters of the sextets and doublets it is possible to conclude that most of the

Fe ions (average on two spectra $\mathrm{A}=91(1) \%$ ) are in the sixfold oxygen coordination in $\mathrm{Fe}^{3+}$ high-spin state. As for the singlet partial spectrum $(\mathrm{A}=9(1) \%)$ it is likely due to an impurity phase containing $\mathrm{Fe}^{3+}$ ions in the four-fold oxygen coordination with cubic symmetry. These data are consistent with earlier reported Mössbauer data obtained at room temperature [28], according to which for low concentrations of Fe doping into the $4 \mathrm{Mn}$ sites (Mn1 (12i), Mn2 (8 h), Mn3 (6f), and Mn4 (2d); using the hexagonal space group $\mathrm{P}_{3} /$ $\mathrm{mcm})$, the Fe atoms were mainly localized in $\mathrm{Mn} 2(8 \mathrm{~h})$ and Mn3 (6f) sites.

Magnetic properties of PMO single crystals have been reported earlier [27]. A paramagnetic behavior was observed above $250 \mathrm{~K}$ followed at lower temperatures by several magnetic transitions. A first anomaly near $160 \mathrm{~K}$ was interpreted as short-range antiferromagnetic ordering within the basal planes of the structure. Then at $67 \mathrm{~K}$ longrange order occurs where, although the overall structure is antiferromagnetic, weak ferromagnetism appears due to 

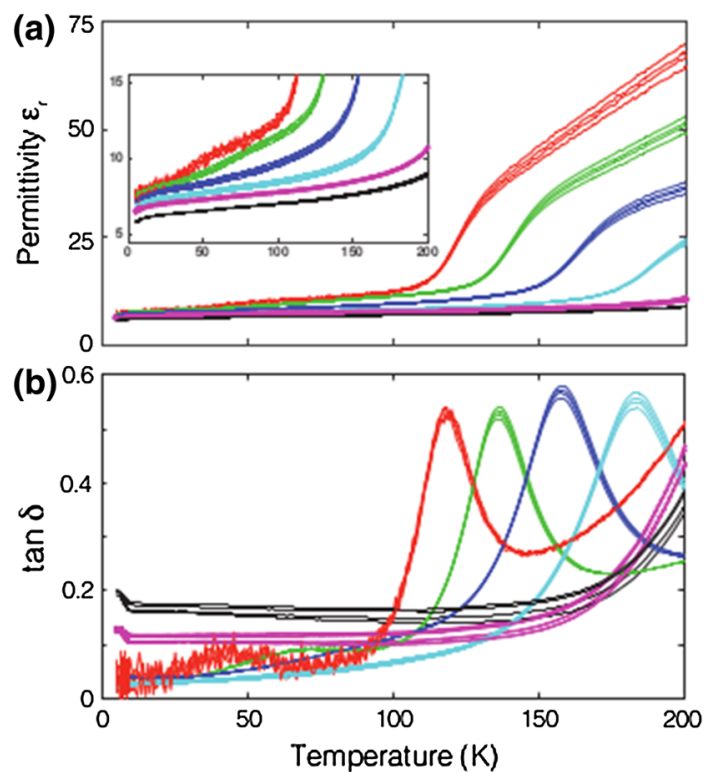

Fig. 4 Temperature dependence of a $\varepsilon$ (inset shows a view focusing on low temperatures), and $\mathbf{b} \operatorname{tg} \delta$, recorded under different magnetic fields. Electrical field $\mathrm{E}$ was applied along the hexagonal c-axis [001]. Magnetic field $\mathrm{H}$ was applied in the hexagonal basal $(a, b)$-plane along [1-10] direction. Groups of curves of similar colors were recorded with frequencies of $100 \mathrm{~Hz}$ (red), $1 \mathrm{kHz}$ (green), $10 \mathrm{kHz}$ (blue), $100 \mathrm{kHz}$ (cyan), $1 \mathrm{MHz}$ (magenta), and $2 \mathrm{MHz}$ (black), under the magnetic fields $\mathrm{H}=0,3,5,7$ and $9 \mathrm{~T}$ (all shown with the same color) (Color figure online)

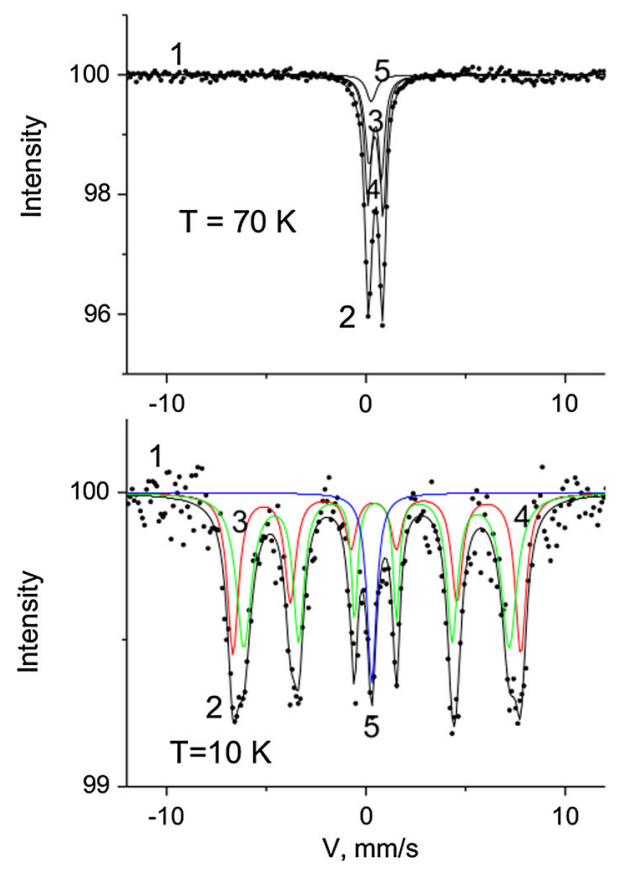

Fig. 5 Mössbauer spectra of the $\mathrm{Pb}_{3}\left(\mathrm{Mn}_{0.98} \mathrm{Fe}_{0.02}\right)_{7} \mathrm{O}_{15}$ sample at $\mathrm{T}=70 \mathrm{~K}($ top $)$ and $10 \mathrm{~K}$ (bottom): 1 data points, 2 total fitting curve, 3,4 and 5 partial

incomplete cancellation of the spins. Finally, at $20 \mathrm{~K}$ another transition interpreted as a reorientation of the spins was reported [27].
Table 3 The parameters of Mössbauer spectra for the $\mathrm{Pb}_{3}\left(\mathrm{Mn}_{1-\mathrm{x}}\right.$ $\left.\mathrm{Fe}_{\mathrm{x}}\right)_{7} \mathrm{O}_{15}, \mathrm{x}=0,02$ sample registered at 10 and $70 \mathrm{~K}$ : isomer shift (IS) with respect to the metallic iron, quadrupole splitting (QS), effective magnetic fields $\mathrm{H}$ at ${ }^{57} \mathrm{Fe}$ nucleus, relative area of partial spectra (A)

\begin{tabular}{llllr}
\hline Temperature, K & IS, mm/s & QS, mm/s & H, kOe & A, \% \\
\hline 10 & $0.47(2)$ & $0.16(4)$ & $449(2)$ & $39(2)$ \\
& $0.50(3)$ & $0.05(4)$ & $414(3)$ & $51(4)$ \\
& $0.29(2)$ & & & $10(2)$ \\
70 & $0.46(2)$ & $0.61(4)$ & - & $41(4)$ \\
& $0.48(2)$ & $0.77(4)$ & - & $51(4)$ \\
& $0.27(0)$ & & & $8(2)$ \\
\hline
\end{tabular}
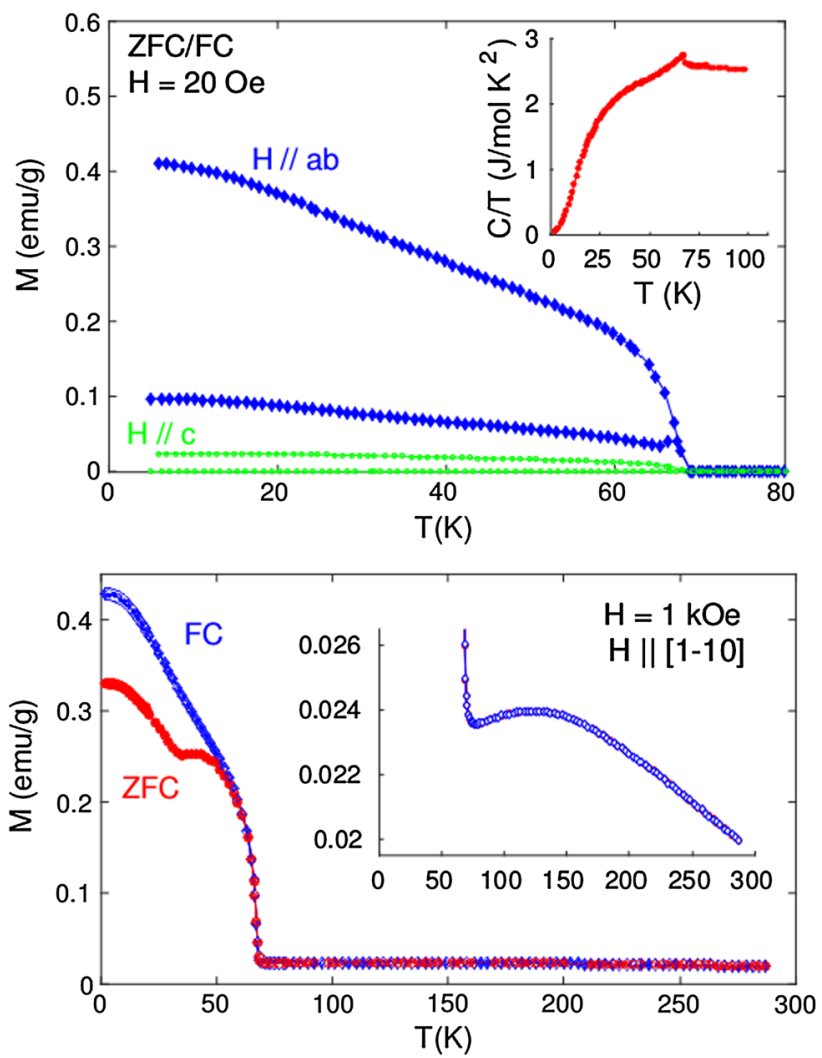

Fig. 6 Top: temperature $\mathrm{T}$ dependence of the zero-field-cooled (ZFC) and field-cooled (FC) magnetization recorded in a small magnetic field applied in and perpendicular to the hexagonal basal $(a, b)$-plane. The inset shows the T-dependence of the heat capacity $\mathrm{C}$, plotted as C/T. Bottom: T-dependence of the ZFC/FC magnetization with $\mathrm{H}=1 \mathrm{kOe}$ applied in the hexagonal basal plane along the [1-10] direction. Inset shows an enlarged view of the high-temperature behavior

The magnetization of our single crystals was measured as a function of temperature and magnetic field (Figs. 6 and 7). Low-field magnetization $M$ and heat capacity data (inset; plotted as $\mathrm{C} / \mathrm{T}$ ) suggest an antiferromagnetic transition near $67 \mathrm{~K}$, with an excess moment in the hexagonal basal plane, similar to that reported in Ref. [27]. The 
remanent magnetization at $2 \mathrm{~K}$ in hysteresis loops amounts to $0.38 \mathrm{emu} / \mathrm{g}$ (c.f. about $0.5 \mathrm{emu} / \mathrm{g}$ in Ref. [27]), which corresponds to a moment of $0.09 \mu_{\mathrm{B}} / \mathrm{f}$.u.

As seen in the M-T data recorded in a larger field $(\mathrm{H}=1 \mathrm{kOe}$, Fig. 6) in the plane along the [1-10] direction, an additional anomaly is observed (broad maximum near $\left.\mathrm{T}^{*} \sim 125 \mathrm{~K}\right)$. This is reminiscent of a similar anomaly in PMO compounds near $160 \mathrm{~K}$ interpreted to be related to short-range antiferromagnetic order and/or charge-orbital order in Refs. [26, 27]. The magnetization does not follow a Curie-Weiss behavior in the range of temperature investigated in Fig. 6. However, a CurieWeiss temperature $\theta_{\mathrm{CW}} \sim 520 \mathrm{~K}$ was reported in a study of magnetic properties up to higher temperatures [27], thus yielding a frustration parameter $\mathrm{f}=\theta_{\mathrm{CW}} / \mathrm{T}_{\mathrm{N}} \sim 8$.

Furthermore, low-temperature behavior of the ZFC magnetization curve recorded in $1 \mathrm{kOe}$ (Fig. 6) and $\mathrm{M}-\mathrm{H}$ hysteresis curves recorded with the magnetic field applied along two different directions in the hexagonal basal plane (Fig. 7) suggest some additional in-plane anisotropy of the excess moment; $\mathrm{M}-\mathrm{H}$ curves recorded with $\mathrm{H} / /[1-10]$ showing a two-step switching behavior [42].
The neutron powder diffraction patterns collected above (70 K) and below $(1.8 \mathrm{~K}) \mathrm{T}_{\mathrm{N}}$ are presented in Fig. 8 . The neutron patterns show additional contributions of intensity for several nuclear peaks below $\mathrm{T}_{\mathrm{N}}$. All observed superstructure magnetic reflections could be indexed with a magnetic propagation vector $\mathbf{k}=(0,0,0)$. This means that no loss in translational symmetry arises from the spin arrangement and identity of the magnetic and chemical cells. The chemical space group of PMO remains Pnma down to $1.8 \mathrm{~K}$ according to our neutron diffraction data. The nine independent magnetic $\mathrm{Mn}$ ions in PMO are distributed on four different sites of the space group Pnma, namely the: $8 \mathrm{~d}(5 \mathrm{Mn}), 4 \mathrm{a}(1 \mathrm{Mn}), 4 \mathrm{~b}(1 \mathrm{Mn})$ and $4 \mathrm{c}(2 \mathrm{Mn})$ sites. This combination of magnetic cations produces 9 independent magnetic sublattices associated with these sites (the coordinates are given in Table SM1 in the Supplemental Materials and a graphical representation is presented in Fig. 9). In order to generate all possible magnetic structures that are compatible with the crystal structure, we have used the program for calculating irreducible representations BASIREPS from the FULLPROF suite [33]. In the framework of the representation analysis, which has been developed by Bertaut [43], it was shown that there are
Fig. 7 Magnetic field dependence of the magnetization at constant temperatures, from $\mathrm{T}=2 \mathrm{~K}$ to $\mathrm{T}=80 \mathrm{~K}$. Magnetic field is applied in the hexagonal basal plane along the [1-10] direction (filled symbols) and [100] direction (open symbols)
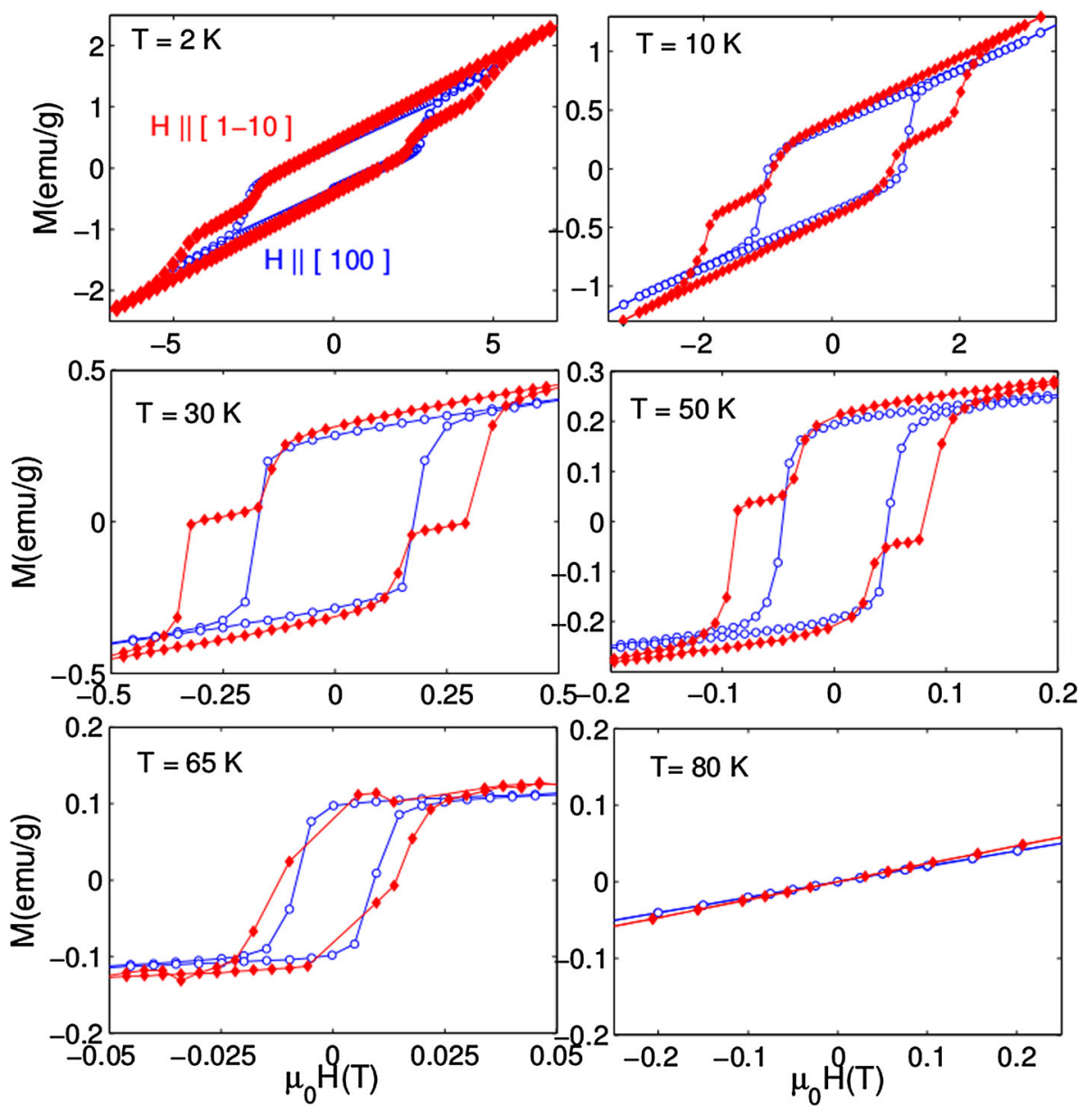
Fig. 8 NPD data and associated Rietveld refinement at $\mathrm{T}=70 \mathrm{~K}(\mathbf{a})$ and $1.8 \mathrm{~K}(\mathbf{b})$. In each panel, upper curves mark data and fit, ticks mark identified reflections and lower curves mark the residue after subtraction of data and fit. At $1.8 \mathrm{~K}$, two rows of ticks are used to mark nuclear and magnetic reflections, respectively. Magnetic peaks in the low temperature data are marked with red arrows in the lower panel (Color figure online)
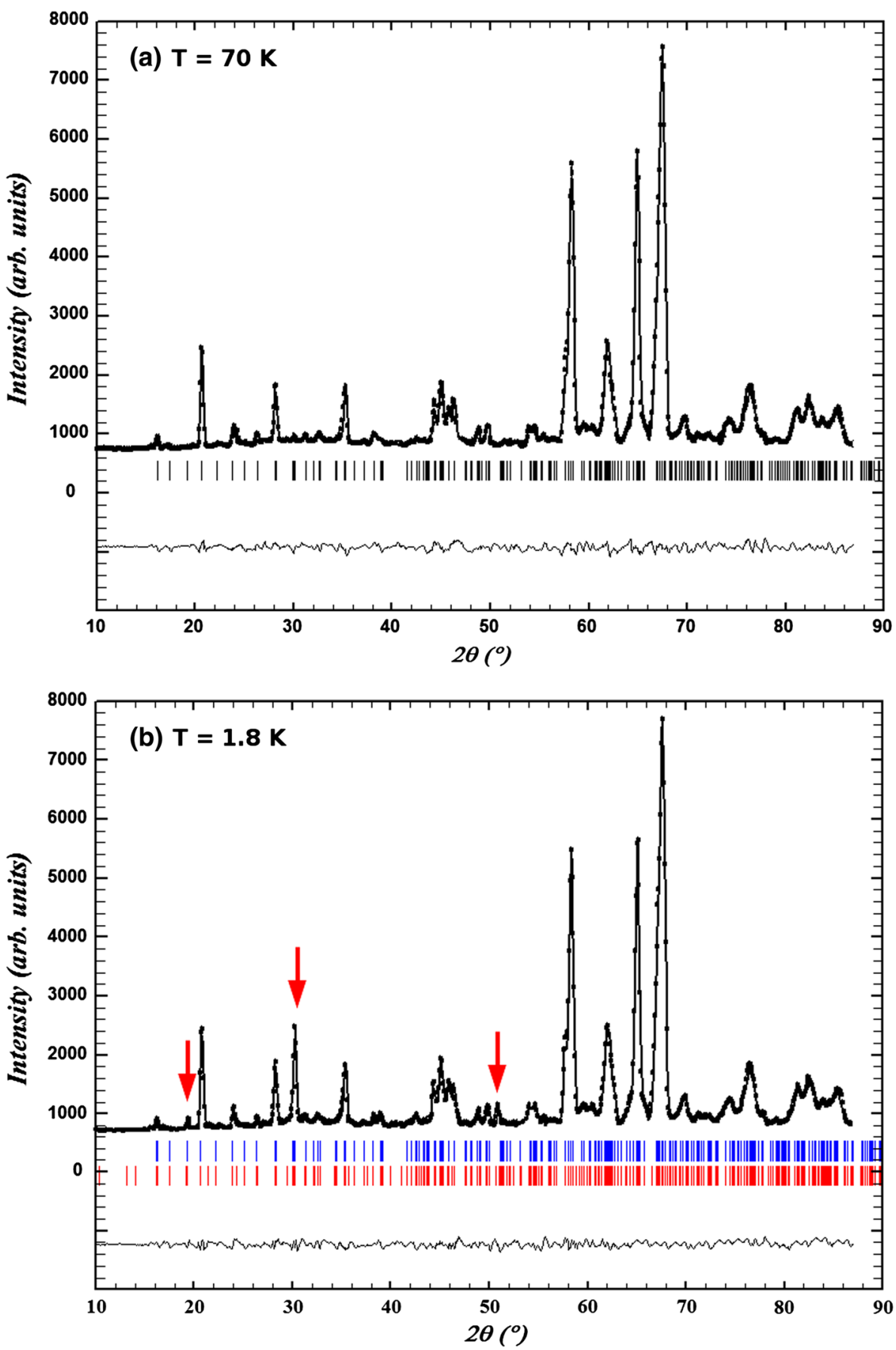

only eight possible one-dimensional irreducible representations $\boldsymbol{G i}(\mathrm{i}=1,2, \ldots, 8)$ of $\mathrm{mmm}$ associated with the propagation vector $\mathbf{k}=(0,0,0)$. They are summarized in Table 4 for the case of the crystallographic space group Pnma. The corresponding magnetic configurations for the $8 \mathrm{~d}, 4 \mathrm{a}, 4 \mathrm{~b}$ and $4 \mathrm{c}$ sites (Table 4 ) are also given. The available positions allow one ferromagnetic $(\boldsymbol{F})$ and three antiferromagnetic $(\boldsymbol{G}, \boldsymbol{C}$ and $\boldsymbol{A}$ ) configurations or modes: $\boldsymbol{F}=\mathrm{S} 1+\mathrm{S} 2+\mathrm{S} 3+\mathrm{S} 4$, and $\boldsymbol{G}=\mathrm{S} 1-\mathrm{S} 2+\mathrm{S} 3-\mathrm{S} 4$,
$\boldsymbol{A}=\mathrm{S} 1-\mathrm{S} 2-\mathrm{S} 3+\mathrm{S} 4, \quad \boldsymbol{C}=\mathrm{S} 1+\mathrm{S} 2-\mathrm{S} 3-\mathrm{S} 4$ where $\mathrm{Sn}$ indicates the spin for the $\mathrm{n}$-fold position. The available set of magnetic reflections allows a preliminary statement to be made concerning the magnetic structure model. Firstly, it was considered that all 9 Mn cations carry magnetic moments and that the basis functions for $8 \mathrm{~d}, 4 \mathrm{a}$, $4 \mathrm{~b}$ and $4 \mathrm{c}$ belong to the same irreducible representation (this is usually the case). The representations $\boldsymbol{G} \mathbf{2}, \boldsymbol{G 4}, \boldsymbol{G 6}$ and $\boldsymbol{G} \boldsymbol{8}$ exclude any magnetic moment on $\mathrm{Mn}$ cations at $4 \mathrm{a}$ 

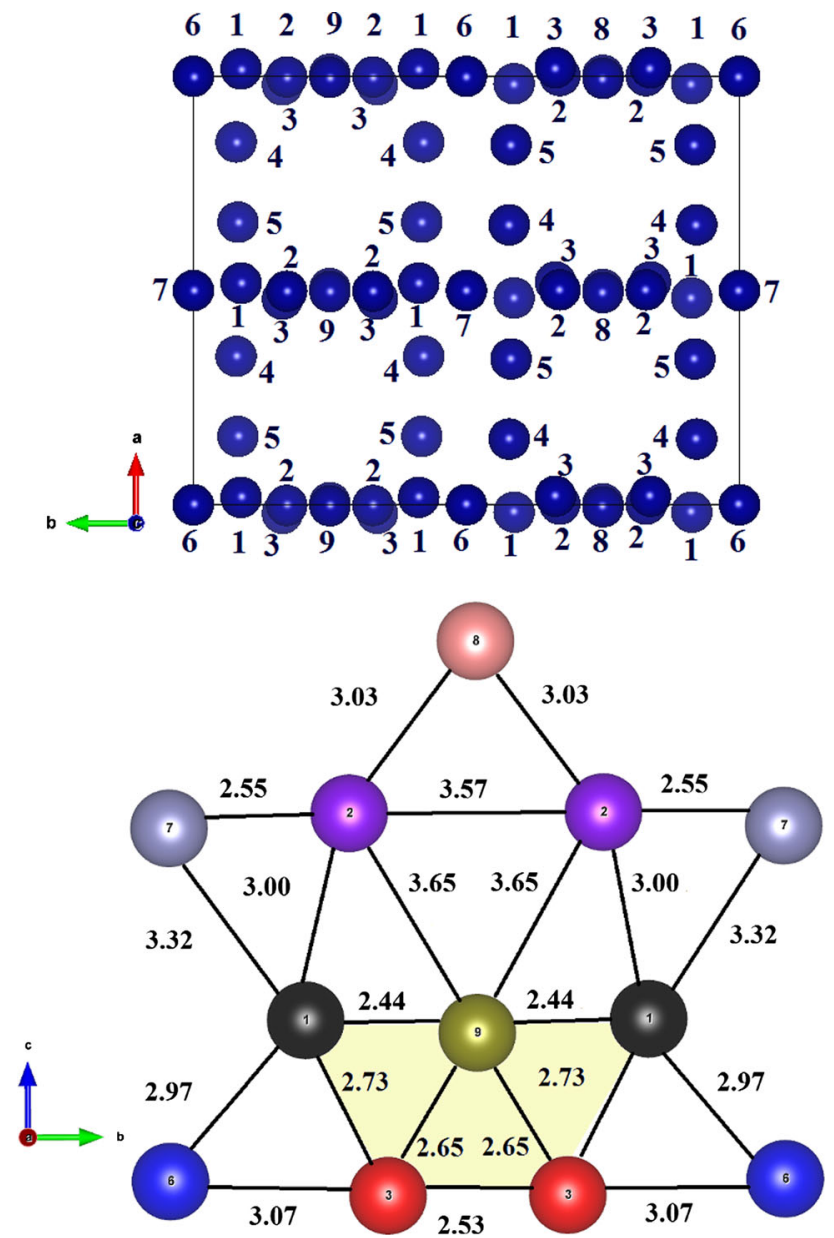

Fig. 9 Identification of the nine inequivalent Mn cation sites in the orthorhombic structure for Top: $(a, b)$ projection, and Bottom: $(b, c)$ projection. In the latter, average $\mathrm{Mn}-\mathrm{Mn}$ distances obtained from polyhedral analysis are indicated in $\AA$. Triangles formed by $\mathrm{Mn}$ cations which are closest to each other are shaded. Lower panel drawn using VESTA [39]

Table $4 \mathrm{x}, \mathrm{y}, \mathrm{z}$-components along a,b, and c axes, and total magnitude of the Mn magnetic moment $\left(\mu_{\mathrm{B}}\right)$ in the magnetic structure of $\mathrm{Pb}_{3} \mathrm{Mn}_{7} \mathrm{O}_{15}$ with $\mathbf{k}=(\mathbf{0 , 0 , 0})$ in the space group Pnma

\begin{tabular}{lrlll}
\hline Cation & X & Y & Z & moment \\
\hline Mn1 & $1.5(4)$ & $0.9(5)$ & $-2.6(4)$ & $3.1(4)$ \\
Mn2 & $-3.6(4)$ & $1.4(5)$ & 0 & $3.9(4)$ \\
Mn3 & $3.5(4)$ & 0 & 0 & $3.5(4)$ \\
Mn4 & $1.4(5)$ & $1.2(5)$ & $2.7(4)$ & $3.3(4)$ \\
Mn5 & $-1.1(5)$ & 0 & $2.4(4)$ & $2.7(4)$ \\
Mn6 & $-4.4(4)$ & 0 & 0 & $4.4(4)$ \\
Mn7 & $-2.5(4)$ & $1.9(5)$ & $-2.0(4)$ & $3.7(4)$ \\
Mn8 & $-1.2(4)$ & 0 & $-2.7(4)$ & $2.9(4)$ \\
Mn9 & $-1.2(5)$ & 0 & 0 & $1.2(5)$ \\
\hline
\end{tabular}

and $4 \mathrm{~b}$ and cannot take part in the solution. The remaining models were hence $\boldsymbol{G} \mathbf{1}(\mathrm{AxGyCz}), \boldsymbol{G} 3(\mathrm{GxAyFz}), \boldsymbol{G 5}$ $(\mathrm{CxFyAz})$ and $\boldsymbol{G} 7$ (FxCyGz). As an additional condition the susceptibility measurements were used, which suggest a canted antiferromagnetic structure and non-compensated magnetic moment in $(b, c)$ plane (i.e. the $(a, b)$ plane in the hexagonal setting considered in magnetic measurements). The lower panel of Fig. 8 shows a typical neutron powder diffraction pattern of PMO measured at $1.8 \mathrm{~K}$ as well as the results of the Rietveld refinement. The nuclear and magnetic structures were refined simultaneously in the antiferromagnetic region. Detailed symmetry analysis of the magnetic structure shows that our experimental NPD pattern corresponds to the magnetic configuration $\mathbf{G} 3$ (GxAyFz).

Good agreement between the observed and calculated intensities of the PMO neutron diffraction patterns (see Fig. 8 as an illustration) indicates the correctness of the model used. The resulting magnetic structure at $1.8 \mathrm{~K}$ is depicted in different views in Fig. 10. If the spin components of 9 different Mn cations belong to various one-dimensional irreducible representations, different magnetic groups would be involved and the real magnetic symmetry could be the intersection of these different magnetic groups. However the case where spin components belong to different representations was outside the scope of our analysis.

The magnetic structure of PMO is characterized by commensurate magnetic ordering of the magnetic moments on nine magnetic sites with mainly antiferromagnetic interactions between edge-sharing Mn-octahedra within partially filled Kagomé planes. The magnetic moments of the different sites are not identical and vary in the frame of 1.2(5)-4.4(4) $\mu_{\mathrm{B}}$ (see also Table 4). However we recognize that this value can also be found to be significantly higher, e.g. $\sim 5.0 \mu_{\mathrm{B}}$. An explanation for the deviation from a higher value could be given by the fact that the magnetic moment is lowered from the covalency of bonds and such covalent effects might be different for existing Mn sites. For PMO, we have found that the magnetic moment of the Mn6 site is located mainly along the a-axis (see Table 4). The Mn1 and Mn4 cations (8d) were found to show basically the same magnetic moment of $\sim 3.2 \mu_{\mathrm{B}}$ but oppositely oriented along the c-axis. The $\mathrm{Mn} 2$ and $\mathrm{Mn} 3$ cations (8d) were found to have similar magnetic moments of $\sim 3.6 \mu_{\mathrm{B}}$ and oppositely oriented along the a-axis. Further analysis shows that the magnetic moments of several of the cations refined along the b-axis are relatively small and there is a clear tendency that the smallest magnetic moment component is found along this direction (see Table 4). The $\mathrm{y}$-component of Mn5, both the $\mathrm{y}$ and $\mathrm{z}$ components of Mn6 and the z-component of Mn2, Mn3 and Mn9 spins were slightly above or within standard deviation values $(0.4-0.5)$. These components were set to zero in the final stage of the refinements. The proposed model of the magnetic structure $\boldsymbol{G} 3(\mathrm{GxAyFz})$ with resulting orientation of overall ferrimagnetic moments along the c-axis agrees 

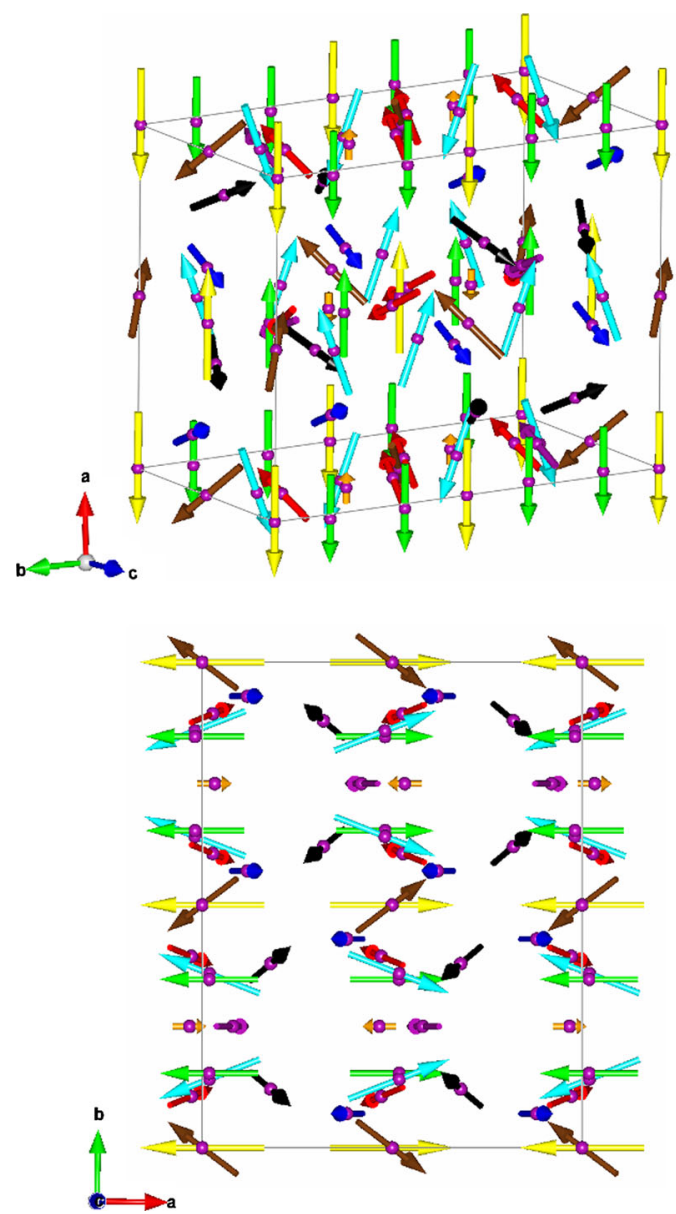

Fig. 10 Different views of the magnetic structure, in which the magnetic moments of the 9 inequivalent $\mathrm{Mn}$ ions are drawn using different colors: Mn1: red; Mn2: cyan; Mn3: green; Mn4: black; Mn5: blue; Mn6: yellow; Mn7: brown; Mn8: pink; Mn9: orange. Note that some magnetic moments oriented perpendicularly to the figure's

well with our magnetic measurements and an earlier study from Volkov et al., who reported that the magnetic easy axis must lie within the (011)-plane (referred to as the hexagonal (001)-plane) [27].

Interplay of ferromagnetic and antiferromagnetic interactions in PMO is plausible due to the numerous inequivalent exchange pathways (see lower panel of Fig. 9) and the competition between antiferromagnetic (superexchange) and ferromagnetic (super exchange and double exchange) interaction [44-46]. Considering these possible exchange interactions, AFM alignment seems to be the most favourable interaction for $\mathrm{Mn}$ spins within the Kagomé planes. However, the triangular arrangements of magnetic moments with each pair coupled antiferromagnetically tend to hinder the system to find a unique ground state, i.e., the geometrical frustration [47, 48]. As seen in the lower panel of Fig. 9, the average Mn-Mn distances vary between 2.44 and $3.65 \AA$; Mn1, Mn3 and Mn9 being
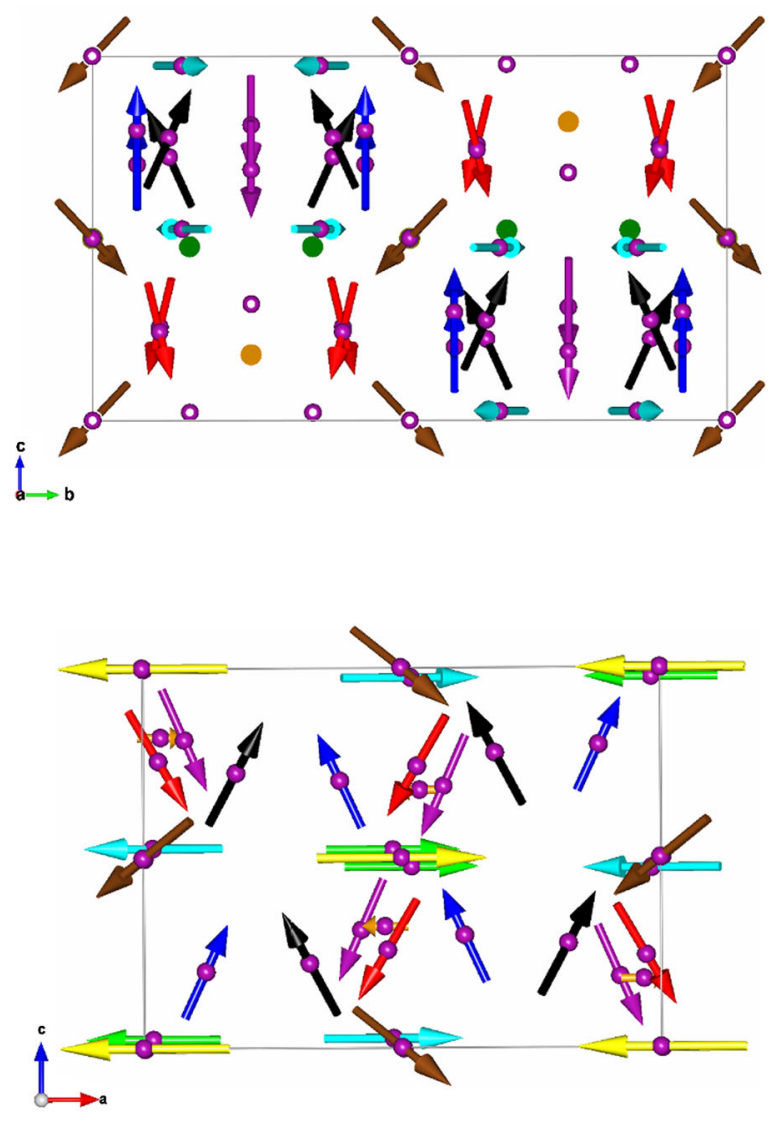

plane may not be visible in all projections, or appear as dots. For example, in $(b, c)$-oriented projection in top right panel, Mn3 moments oriented "down" along the a direction, do not appear in the figure, while those oriented "up" along the a direction appear as green dots (Color figure online)

the closest, as indicated by the shaded Mn-triangles in Fig. 9. The chemical formula $\mathrm{Pb}_{3} \mathrm{Mn}_{7} \mathrm{O}_{15}$ indicates a mixed-valence system with coexisting of $\mathrm{Mn}^{3+}$ and $\mathrm{Mn}^{4+}$ ( $3 \mathrm{Mn}^{4+}$ and $4 \mathrm{Mn}^{3+}$ per formula unit). The distribution of $\mathrm{Mn}^{3+} / \mathrm{Mn}^{4+}$ between possible sites is influenced by the lone electron pairs of $\mathrm{Pb}^{2+}$ cations. Bond valence calculations (see Table 2) indicate some charge ordering of $\mathrm{Mn}^{3+}$ and $\mathrm{Mn}^{4+}$ cations for PMO but a complete description of this charge ordering is still under investigation $[26,28]$. Considering the $\mathrm{d}^{4}$ electronic configuration of $\mathrm{Mn}^{3+}$ and associated Jahn-Teller distortions, it was suggested that the $\mathrm{Mn}^{3+}$ ions would occupy the $\mathrm{Mn} 4$ and Mn5 positions while the $\mathrm{Mn}^{4+}$ ions would occupy the less distorted Mn6 and Mn7 positions [16]. Such a distribution gives a $\mathrm{Mn}^{3+} / \mathrm{Mn}^{4+}$ ratio of $4: 3$ which is equal to the value obtained when assuming that the unit cell has a zero electric charge. Another argument supporting this model was that the oxygen octahedra around $\mathrm{Mn}^{4+}$ ions are 
almost undistorted. From Table 2 it is clear that all of the Mn-octahedra are differently distorted. However, it is not straightforward to determine the $\mathrm{Mn}^{3+}$ and $\mathrm{Mn}^{4+}$ sites from crystal data alone. One possible way is to place $\mathrm{Mn}^{4+}$ at sites with shortest average $\mathrm{Mn}-\mathrm{O}$ distance around them.

\section{Conclusions}

The structural, magnetic, dielectric properties of $\mathrm{Pb}_{3} \mathrm{Mn}_{7}$ $\mathrm{O}_{15}$ have been investigated in detail. Neutron powder diffraction on crushed single crystals is used to determine the low-temperature antiferromagnetic magnetic structure. Mn has average valence +3.4 in the material, and is distributed onto 9 independent octahedral sites in a specific orthorhombic structure with partly filled Kagomé layers connected by ribbons of edge-sharing $\mathrm{MnO}_{6}$ octahedra and intercalated $\mathrm{Pb}^{2+}$ cations. Structural distortion originates from lone-electron pair of $\mathrm{Pb}^{2+}$ cations and from the presence of particular charge ordering of $\mathrm{Mn}^{3+}$ and $\mathrm{Mn}^{4+}$ cations in the octahedral sites, $57 \%$ of which are occupied by the Jahn-Teller active $\mathrm{Mn}^{3+}$ cations. We discuss in detail the associated geometrical frustration, and complex interplay between structural distortions and magnetic interaction paths in $\mathrm{Pb}_{3} \mathrm{Mn}_{7} \mathrm{O}_{15}$. It is unclear whether the oxygen content may influence the phase stability in PMO, as the oxygen index $\delta$ was slightly larger than zero for single-crystals with hexagonal space group, and slightly lower than zero for the powders with orthorhombic one.

Acknowledgments Financial support from the Swedish Research Council (VR), the Göran Gustafsson Foundation, the Swedish Foundation for International Cooperation in Research and Higher Education (STINT), the Russian Foundation for Basic Research, and the FIRST program on "Quantum Science on Strong Correlation" from JSPS, Japan, is gratefully acknowledged. M.H. thanks AnnaMaria Lundin Foundation and Hans Werthén Fonden.

Open Access This article is distributed under the terms of the Creative Commons Attribution 4.0 International License (http://crea tivecommons.org/licenses/by/4.0/), which permits unrestricted use, distribution, and reproduction in any medium, provided you give appropriate credit to the original author(s) and the source, provide a link to the Creative Commons license, and indicate if changes were made.

\section{References}

1. C.N.R. Rao, B. Raveau (eds.), Colossal Magnetoresistance (Charge Ordering and Related Properties of Manganese Oxides, World Scientific, 1998)

2. Y. Tokura, Y. Tomioka, J. Magn. Magn. Mater. 200, 1 (1999)

3. Y. Tokura, N. Nagaosa, Science 288, 462 (2000)

4. K.H. Kim, M. Uehara, V. Kiryukhin, S.W. Cheong, in Colossal Magnetoresistive Manganites, ed. by T. Chatterji (Kluwer-Academic, Dordrecht, 2004)
5. D. Choudhury, P. Mandal, R. Mathieu, A. Hazarika, S. Rajan, A. Sundaresan, U.W. Waghmare, R. Knut, O. Karis, P. Nordblad, D.D. Sarma, Phys. Rev. Lett. 108, 127201 (2012)

6. T. Kimura, S. Kawamoto, I. Yamada, M. Azuma, M. Takano, Y. Tokura, Phys. Rev. B 67, 180401(R) (2003)

7. T. Kimura, T. Goto, H. Shintani, K. Ishizaka, T. Arima, Y. Tokura, Nature 426, 55 (2003)

8. C. Daniel, J.O. Besenhard, Handbook of Battery Materials, vol. 2, 2nd edn. (Wiley-VCH Verlag GmbH\&Co, USA, 2011)

9. J.E. Post, D.L. Bish, Amer. Miner. 74, 913 (1989)

10. B.I. Al'shin, D.N. Atrov, L.N. Baturov, JETP Lett. 22, 214 (1975)

11. B.I. Al'shin, R.V. Zorin, L.A. Drobyshev, S.V. Stepanishchev, Soviet. Phys. Crystal. 3, 489 (1972)

12. A.A. Bush, A.V. Titov, B.I. Al'shin, YuN Venevtsev, Russ. J. Inorg. Chem. 22, 1211 (1977)

13. B. Latourrette, M. Devalette, F. Js Guillen, C. Fouassier, Mat. Res. Bull 13, 567 (1978)

14. P.B. Darriet, M. Devalette, B. Latourrette, Acta Cryst. B 34, 3528 (1978)

15. Y. Le Page, L.D. Calvert, Acta Cryst. C 40, 1787 (1984)

16. J.C.E. Rasch, D.V. Sheptyakov, J. Schefer, L. Keller, M. Boehm, F. Gozzo, N.V. Volkov, K.A. Sablina, G.A. Petrakovskii, H. Grimmer, K. Conder, J.F. Löffler, J. Solid State Chem. 182, 1188 (2009)

17. N.V. Volkov, L.A. Solovyov, E.V. Eremin, K.A. Sablina, S.V. Misjul, M.S. Molokeev, A.I. Zaitsev, M.V. Gorev, A.F. Bovin, N.V. Mihashenok, Phys. B 407, 689 (2012)

18. E.A. Dalchiele, S. Cattarin, M. Musiani, U. Casellato, P. Guerriero, J. Appl. Electrochem. 30, 117 (2000)

19. A.M. Abakumov, J. Hadermann, A.A. Tsirlin, H. Tan, J. Verbeeck, E.V. Dikarev, R.V. Shpanchenko, E.V. Antipov, J. Solid State Chem. 182, 2231 (2009)

20. A. Teichert, Hk. Muller-Buschbaum, Z. Anorg, Allg. Chem 598/ 599, 319 (1991)

21. S.A.J. Kimber, J.P. Attfield, J. Mater. Chem. 17, 4885 (2007)

22. C. Bougerol, M.F. Gorius, I.E. Grey, J. Solid State Chem. 169, 131 (2002)

23. K. Oka, M. Azuma, S. Hirai, A.A. Belik, H. Kojitani, M. Akaogi, M. Takano, Y. Shimakawa, Inorg. Chem. 48, 2285 (2009)

24. J. Hadermann, A.M. Abakumov, A.A. Tsirlin, V.P. Filonenko, J. Gonnissen, H. Tan, J. Verbeeck, M. Gemmie, E.V. Antipov, H. Rosner, Ultramicrosc. 110, 881 (2010)

25. T. Locherer, R. Dinnebier, R.K. Kremer, M. Greenblatt, M. Jansen, J. Solid State Chem. 190, 277 (2012)

26. N.V. Volkov, K.A. Sablina, E.V. Eremin, P. Boni, V.R. Shah, I.N. Flerov, A. Kartashev, J.C.E. Rasch, M. Boehm, J. Schefer, J. Phys.: Condens. Matter 20, 445214 (2008)

27. N.V. Volkov, K.A. Sablina, O.A. Bayukov, E.V. Eremin, G.A. Petrakovskii, D.A. Velikanov, A.D. Balaev, A.F. Bovina, P. Boni, E. Clementyev, J. Phys.: Condens. Matter 20, 055217 (2008)

28. S.A.J. Kimber, J. Phys.: Condens. Matter 24, 186002 (2012)

29. N.V. Volkov, E.V. Eremin, O.A. Bayukov, K.A. Sablina, L.A. Solov'ev, D.A. Velikanov, N.V. Mikhashenok, E.I. Osetrov, J. Schefer, L. Keller, M. Boehm, J. Magn. Magn. Mater. 342, 100 (2013)

30. N.V. Volkov, E.V. Eremin, K.A. Sablina, N.V. Sapronova, J. Phys.: Condens. Matter 22, 375901 (2010)

31. R.E. Marsh, F.H. Herbstein, Acta. Cryst. B 39, 280 (1983)

32. D. Holtstam, B. Lindquist, M. Johnsson, R. Norrestam, Can. Miner. 29, 347 (1991)

33. N.K. Volkov, E.V. Eremin, O.A. Bayukov, K.A. Sabina, Book of Abstracts of the International Workshop on Phase Transitions and Inhomogeneous States in Oxides (Kazan, Russia, 2015)

34. J. Rodriguez-Carvajal, Phys. B 192, 55 (1993)

35. T.B. Zunic, I. Vickovic, J. Appl. Cryst. 29, 305 (1996) 
36. V.S. Rusakov, Messbauerovskaya spektroskopiya lokal'no neodnorodnykh sistem [Mössbauer spectroscopy of locally inhomogeneous systems]. Almaty, INP NNC RK Publ. (2000), p. 431. ISBN 9965-9111-2-6

37. D. Orobengoa, C. Capillas, M.I. Aroyo, J.M. Perez-Mato, J. Appl. Crystallogr. 42, 820 (2009)

38. J.M. Perez-Mato, D. Orobengoa, M.I. Aroyo, Acta Crystallogr. A 66, $558(2010)$

39. K. Momma, F. Izumi, VESTA 3 for three-dimensional visualization of crystal, volumetric and morphology data. J. Appl. Crystallogr. 44, 1272 (2011)

40. S.A. Arrhenius, Z. Phys, Chem. 4, 96 (1889)
41. A.K. Jonscher, Dielectric Relaxation in Solids (Chelsea Dielectric Press, London, 1983)

42. W.-C. Lin, C.B. Wu, P.J. Hsu, H.Y. Yen, Z. Gai, L. Gao, J. Shen, M.-T. Lin, J. Appl. Phys. 108, 034312 (2010). and references therein

43. E.F. Bertaut, Acta Cryst. A 24, 217 (1968)

44. J.B. Goodenough, Phys. Rev. 100, 564 (1955)

45. J.B. Goodenough, Magnetism and the Chemical Bond (Wiley, New York, 1963)

46. J. Kanamori, J. Phys. Chem. Solids 10, 87 (1959)

47. A.P. Ramirez, Annu. Rev. Mater. Sci. 24, 453 (1994)

48. J.E. Greedan, J. Mater. Chem. 11, 37 (2001) 